\title{
Nonlinear Optical Rectification of Tuned Quantum Dots Under the Action of a Vertical Magnetic Field
}

\author{
Zhuang Zhao ( $\nabla 22374751 @ q q . c o m$ ) \\ Anhui University of Science and Technology
}

\section{Research Article}

Keywords: Tuned quantum dots, Nonlinear optical rectification, Eigenfunction, Density matrix theory

Posted Date: November 29th, 2021

DOI: https://doi.org/10.21203/rs.3.rs-1083830/v1

License: (9) This work is licensed under a Creative Commons Attribution 4.0 International License. Read Full License 


\title{
Nonlinear Optical Rectification of Tuned Quantum Dots under the Action of a Vertical Magnetic Field
}

\begin{abstract}
Zhuang Zhao
Abstract: The effects of tuned quantum dots (QD) on the optical rectification (OR) coefficient under the action of the external magnetic field, hydrostatic pressure, temperature and quantum dot radius is theoretically studied in detail. The tuned quantum dots are subjected to a uniform magnetic field perpendicular to the structure plane. Within the framework of effective mass approximation and parabolic approximation, the energy level and wave function are derived, and the nonlinear optical rectification coefficients are calculated by the compact density matrix method and iterative method. Numerical results show that under different constraint parameters, the resonance peak of the OR coefficient moves in the direction of high energy or low energy, that is, red shift or blue shift. At the same time, the peak value of the OR coefficient will increase or decrease with the change of the parameters.
\end{abstract}

Keywords: Tuned quantum dots; Nonlinear optical rectification; Eigenfunction; Density matrix theory

\section{Introduction}

Since the introduction of the new concept of quantum dots in the 1990s, it has caused extensive research by scientific researchers, and its essence is a nano-level semiconductor. Applying a certain electric field or light pressure to this nano-semiconductor material will emit light of a specific frequency. The frequency of the emitted light will change with the size of the semiconductor, such as the shape and size of quantum dots, etc. This nano-semiconductor has the characteristic of limiting electrons and electron holes, called the quantum confinement effect. This characteristic accords with the characteristics of atoms; therefore, quantum dots are also called quantum dot atoms. People are conducting extensive research on the new physical phenomena in this restricted structure. In the past ten years, people have been interested in the nonlinear optical properties of semiconductor quantum systems, such as quantum wells, quantum wires, quantum dots and superlattices, among which quantum dots occupy a large proportion. Quantum dots have good light stability, broad excitation spectrum and narrow emission spectrum, making them extremely useful in fluorescent labelling. At the same time, quantum dots also have the advantages of large Stokes shift and long fluorescence lifetime, which is conducive to detecting fluorescence spectral signals. Due to its unique characteristics, it has made great contributions to energy, solar cells, medicine and biology. It has become the object of intensive research in the past 40 years.

Quantum confinement fundamentally changes the electronic and optical properties of spherical quantum dots. Early experiments show that the confinement potential in quantum contact is mainly parabolic. Parabolic confinement is more appropriate when the quantum dots are prepared by etching, ion implantation or electrostatic gates. The parabolic confinement potential can accommodate various resonances, and the nonlinear polarization is enhanced due to the constant spacing of the energy levels, which is conducive to light absorption. Subsequently, people have conducted various optical studies on parabolic and semi-parabolic quantum dots. In recent years, Zhuang Zhao, Faculty of Technology, College of Mechanics and Photoelectric Physics, Anhui University of Science and Technology, 232001 Huainan, China, Email:522374751@qq.com. 
many researchers have conducted many theoretical studies on the nonlinear optical properties of different quantum dots. Bin $\mathrm{Li}$ et al. [9] studied the nonlinear optical rectification of parabolic quantum dots in the presence of electric and magnetic fields; Zhi-Hai Zhang et al. [10] studied the light absorption coefficient and refractive index changes of parabolic quantum dots under the action of electric and magnetic fields; L. Bouzai ene et al. [11] studied the influence of static pressure and temperature on the nonlinear optical rectification of $\mathrm{AlGaAs} / \mathrm{GaAs}$ quantum dot lens shapes; Ganguly et al. [12] studied the presence and absence of Gaussian white noise, Under the combined influence of hydrostatic pressure and temperature, the characteristics of optical rectification, second harmonic generation and third harmonic generation of impurity-doped quantum dots; R. Khordad et al. [13] studied the effect of magnetic field on the linearity of parabolic quantum dots. The influence of nonlinear optical properties; Suman Dahiya et al. [14] studied the effects of temperature and hydrostatic pressure on the optical rectification of the exciton system in semi-parabolic quantum dots; Liangcheng Zhang et al. [15] theoretically studied the use of compact density matrix method and iterative method to obtain the second harmonic generation coefficient in the z-direction under the action of an external electric and magnetic field. However, the nonlinear optical rectification in tuned quantum dots under the action of a vertical magnetic field, hydrostatic pressure and temperature have not been studied. Therefore, in this article, we will theoretically study the influence of factors such as the vertical magnetic field and hydrostatic pressure in the tuned quantum dots on the optical rectification coefficient.

Quantum confinement fundamentally changes the electronic and optical properties of spherical quantum dots. Early experiments show that the confinement potential in quantum contact is mainly parabolic. Parabolic confinement is more appropriate when the quantum dots are prepared by etching, ion implantation or electrostatic gates. The parabolic confinement potential can accommodate various resonances, and the nonlinear polarization is enhanced due to the constant spacing of the energy levels, which is conducive to light absorption. Subsequently, people have conducted Under the impetus of these works, the influence of the modified Gaussian potential modulated parabolic inverse square confinement potential on the nonlinear optical rectification coefficient of the two-dimensional single-electron quantum dot system is theoretically studied. In the first section of Chapter 2, the time-independent Schrödinger equation is numerically solved based on the effective mass approximation and the parabolic band approximation, and the wave function and energy level are obtained; In the second section, a simple analytical formula for the OR coefficient is derived using the compact density matrix method and iterative method. In the third chapter, plotting, analysis and discussion are carried out based on the numerical results. The results show that factors such as vertical magnetic field, hydrostatic pressure, and temperature significantly affect the OR coefficient. Chapter 4 gives a summary.

\section{Theory and model}

\subsection{Wave function and energy eigenvalue}

This research is based on a theoretical model composed of a parabolic inverse square bound potential electron with Gaussian correction and a magnetic field perpendicular to its plane. The bound potential $V_{1}(r)$ in this model is composed of parabolic and inverse square potential functions, and its form is [16-18] 


$$
V_{1}(r)=\frac{1}{2} m(P, T)^{*} \omega_{0}^{2} r^{2}+\frac{\hbar^{2}}{2 m(P, T)^{*}} \frac{\xi}{r^{2}}
$$

Where $\xi$ is a dimensionless parameter, $\omega_{0}$ is the harmonic constraint frequency, $\hbar$ is the reduced Planck constant, $m(P, T)^{*}$ is the effective mass related to hydrostatic pressure $\mathrm{P}$ and temperature $\mathrm{T}$. The expression is as follows

$$
\frac{m_{0}}{m(P, T)^{*}}=1+E_{p}^{\Gamma}\left[\frac{2}{E_{g}^{\Gamma}(P, T)}+\frac{1}{E_{g}^{\Gamma}(P, T)+\Delta_{0}}\right]
$$

In the above formula, $m_{0}$ 为 is the free electron mass, $E_{p}^{\Gamma}$ is the energy associated with the momentum matrix element and equal to $7.51 \mathrm{eV}$, and $\Delta_{0}$ is the energy of the spin-orbit splitting. For GaAs materials, $E_{g}^{\Gamma}(P, T)$ is the energy gap function related to static pressure $\mathrm{P}(\mathrm{kbar})$ and temperature $\mathrm{T}(\mathrm{K})$, and the analytical form is as follows $[19,20]$

$$
E_{g}^{\Gamma}(P, T)=E_{g}^{\Gamma}(0, T)+1.26 \times 10^{-2} \times P-3.77 \times 10^{-5} \times P^{2}
$$

With $E_{g}^{\Gamma}(0, T)=\left[1.519-\left(5.405 \times 10^{-4} T^{2}\right) /(T+204)\right]$.

The modified Gaussian potential can be expressed by the following expression

$$
V_{2}(r)=-V_{0} \exp \left[-\left(\frac{r}{R_{0}}\right)^{q}\right]
$$

In the above formula, $V_{0}$ and $R_{0}$ are the depth and stretching range of the constrained potential, respectively, where $R_{0}$ can be considered as the radius of the quantum dot, and suppose $\mathrm{q}=2$, and $r / R_{0} \ll 1$. Therefore, the Gaussian potential can be written as the anisotropic harmonic oscillator potential as $[21,22]$

$$
V_{2}(r)=-V_{0} \exp \left[-\left(\frac{r}{R_{0}}\right)^{2}\right]
$$

So the total effective potential is the sum of two parts

$$
V(r)=V_{1}(r)+V_{2}(r)
$$

With the effective mass approximation, the eigenstate of the Hamiltonian of the stationary electron in the system is

$$
H=\frac{1}{2 m(P, T)^{*}}\left[-i \hbar \nabla+\frac{e}{c} \vec{A}\right]^{2}+V(r)
$$

The constant $\mathrm{c}$ is the speed of light, and $\vec{A}$ represents the vector potential, which can be expressed as $\vec{A}=(0, B r / 2,0)$ using the Coulomb criterion, so the above Hamiltonian can also be expressed as

$$
\begin{gathered}
H=\frac{1}{2 m(P, T)^{*}}\left(\frac{\partial^{2}}{\partial r^{2}}+\frac{1}{r} \frac{\partial}{\partial r}+\frac{1}{r^{2}} \frac{\partial^{2}}{\partial \theta^{2}}\right)+\frac{1}{2} m(P, T)^{*}\left(\omega_{0}^{2}+\frac{\omega_{c}^{2}}{4}\right) r^{2}+\frac{V_{0} r^{2}}{R_{0}^{2}}-V-i \hbar \frac{\omega_{c}}{2} \frac{\partial}{\partial \theta}+ \\
\frac{\hbar^{2}}{2 m(P, T)^{* 2}} \frac{\xi}{r^{2}}
\end{gathered}
$$


Among them, $\Omega^{2}=\left(\omega^{2}+\frac{\omega_{c}^{2}}{4}+\frac{2 V_{0}}{m^{*} R_{0}^{2}}\right)$ is the effective frequency, and $\omega_{c}=e B / m^{*} c$, t This Hamiltonian model fully expresses two-dimensional quantum dots with single-carrier electrons, and its confinement potential conforms to the transverse electrostatic confinement of electrons on the $x y$ plane. The time-independent Schrödinger equation can be expressed as

$$
\mathrm{H} \Psi=E \Psi
$$

Among them, $\Psi$ is the two-dimensional eigenstate of a pure quantum dot, $E$ is the corresponding eigenvalue, and the above formula is often written as

$$
\Psi(r, \theta)=R(r) \frac{e^{i m \theta}}{\sqrt{2 \pi}}
$$

In the formula, $m$ is the magnetic quantum number and is an integer. Substituting the above equation and the Hamiltonian expression into Schrodinger's equation (9), and eliminating the same terms on the left and right sides of the equation, we get [23-25]

$$
\left[-\frac{\hbar^{2}}{2 m^{*}}\left(\frac{\partial^{2}}{\partial r^{2}}+\frac{1}{r} \frac{\partial}{\partial r}-\frac{m^{2}}{r^{2}}\right)+\frac{1}{2} m^{*} \Omega^{2} r^{2}-V_{0}+\frac{m \omega_{c} \hbar}{2}+\frac{\hbar^{2}}{2 m^{*}} \frac{\xi}{r^{2}}\right] R(r)=E R(r)
$$

For the convenience of calculation, further substitutions are made, so that

$$
R(r)=\frac{\chi(r)}{\sqrt{r}}
$$

At this point, (11) becomes

$$
\frac{d^{2} \chi(r)}{d r^{2}}+\left[\frac{2 m^{*} \lambda}{\hbar^{2}}-\frac{m^{*} \Omega^{2} r^{2}}{\hbar^{2}}-\frac{l(l+1)}{r^{2}}\right] \chi(r)=0
$$

Among them, $\lambda=E^{0}+V_{0}-m \hbar \omega_{c} / 2, l(l+1)=m^{2}+\xi-1 / 4, l$ is always a positive number, you can define $l=\sqrt{m^{2}+\xi}-1 / 2$. For further solving, let $r^{2}=\Omega_{1}^{2} z, l_{m}=\sqrt{m^{2}+\xi} /$ $2+1 / 4, \Omega_{1}=\sqrt{\hbar / m^{*} \Omega}$ and $\Omega_{2}=\lambda / 2 \hbar \Omega$, After substituting formula (13), we get

$$
\frac{d^{2} \chi(z)}{d z^{2}}+\frac{1}{2 z} \frac{d \chi(z)}{d z}-\left[\frac{1}{4}+\frac{l_{m}\left(l_{m}-\frac{1}{2}\right)}{z^{2}}-\frac{\Omega_{2}}{z}\right] \chi(z)=0
$$

Since the wave function has asymptotic properties at the origin and infinity, it can be assumed

$$
\chi(z)=z^{l m} e^{-\frac{z}{2}} W(z)
$$

Substituting the above formula into equation (14), we can get

$$
z \frac{d^{2} W(z)}{d z^{2}}+\left[\left(2 l_{m}+\frac{1}{2}\right)-z\right] \frac{d W(z)}{d z}-\left(l_{m}+\frac{1}{4}-\Omega_{2}\right) W(z)=0
$$

Let $\left(2 l_{m}+\frac{1}{2}\right)=b$ and $\left(l_{m}+\frac{1}{4}-\Omega_{2}\right)=a$ further simplify, the original equation becomes

$$
z \frac{d^{2} W(z)}{d z^{2}}+(b-z) \frac{d W(z)}{d z}-a(z)=0
$$


The form of the equation at this time conforms to the well-known Kummer differential equation, and the solution of this equation is a confluence hypergeometric function. In order to make $\chi(z)$ finite, let $a=-n$, and we can get

$$
W(-n, b ; z)=\frac{\Gamma(1+n)}{\Gamma(b+n)} \Gamma(b) L_{n}^{(b-1)}(z)
$$

Where $\Gamma(x)$ is the Euler gamma function, and $L_{n}^{(b-1)}(z)$ is the related Laguerre polynomial, so

$$
\chi(z)=z^{l m} e^{-\frac{z}{2}} \frac{\Gamma(1+n)}{\Gamma(b+n)} \Gamma(b) L_{n}^{(b-1)}(z)
$$

Simultaneous equations (12) and equation (19) can be obtained

$$
R(r)=\frac{r^{2 l_{m}-\frac{1}{2}}}{\Omega_{1}^{2 l_{m}}} e^{\frac{-r^{2}}{2 \Omega_{1}^{2}}} \frac{\Gamma(1+n)}{\Gamma(b+n)} \Gamma(b) L_{n}^{\left(2 l_{m}-\frac{1}{2}\right)}\left(\frac{r^{2}}{\Omega_{1}^{2}}\right)
$$

Therefore, the normalized intrinsic wave function of the quantum dot system can be obtained from equation (10) as

$$
\Psi_{m n}(r, \theta)=\sqrt{\frac{n !}{\pi \Gamma\left(2 l_{m}+n+\frac{1}{2} \Omega_{1}^{\left(4 l_{m}+1\right)}\right)}} r^{2 l_{m}-\frac{1}{2}} e^{\frac{-r^{2}}{2 \Omega_{1}^{2}}} L_{n}^{\left(2 l_{m}-\frac{1}{2}\right)}\left(\frac{r^{2}}{\Omega_{1}^{2}}\right) e^{i m \theta}
$$

With

$$
\begin{aligned}
& a=-n \\
& n=-l_{m}-\frac{1}{4}+\Omega_{2} \\
& n=-l_{m}-\frac{1}{4}+\frac{E+V_{0}-\frac{m \hbar \omega_{c}}{2}}{2 \hbar \Omega} E=\left(2 n+2 l_{m}+\frac{1}{2}\right) \hbar \Omega+\frac{m \hbar \omega_{c}}{2}-V_{0}
\end{aligned}
$$

The energy eigenvalue of the quantum dot system is

$$
E_{m n}=\left(2 n+\sqrt{m^{2}+\xi}+1\right) \hbar \Omega+\frac{m \hbar \omega_{c}}{2}-V_{0}
$$

\subsection{Optical rectification coefficient}

Assuming that the system is excited by an electromagnetic field, the electromagnetic field can be expressed as [26-28]

$$
E(t)=E_{0} \cos (\omega t)=\tilde{E} e^{i \omega t}+\tilde{E} e^{-i \omega t}
$$

The evolution of the density matrix operator $\rho$ follows the following Schrödinger equation that varies with time

$$
\frac{\partial \rho_{i j}}{\partial t}=\frac{1}{i \hbar}\left[H_{0}-q z E(t), \rho\right]_{i j}-\Gamma_{i j}\left(\rho-\rho^{(0)}\right)_{i j}
$$

Where $\rho^{(0)}$ is the undisturbed density matrix operator, $\Gamma_{i j}$ is the relaxation rate, and $H_{0}$ is the Hamiltonian of the system without electromagnetic field. The above formula can be calculated using an iterative method [29]

$$
\rho(t)=\sum_{n} \rho^{(n)}(t)
$$




$$
\frac{\partial \rho_{i j}^{(n+1)}}{\partial t}=\frac{1}{i \hbar}\left\{\left[H_{0}, \rho^{(n+1)}\right]_{i j}-i \hbar \Gamma_{i j}\left(\rho-\rho^{(n+1)}\right)_{i j}\right\}-\frac{1}{i \hbar}\left[q Z, \rho^{(n)}\right]_{i j} E(t)
$$

The electrical polarization of the quantum dot due to the electromagnetic field $E(t)$ can be expressed as

$$
P^{(n)}(t)=\left(\varepsilon_{0} \chi^{(1)} \tilde{E} e^{i \omega t}+\varepsilon_{0} \chi_{0}^{(2)}|\tilde{E}|^{2}+\varepsilon_{0} \chi_{2 \omega}^{(2)} \tilde{E}^{2} e^{2 i \omega t}\right)+c . c .
$$

Where $\chi^{(1)} 、 \chi_{0}^{(2)} 、 \chi_{2 \omega}^{(2)}$ are linear susceptibility, optical rectification, and second harmonic generation, respectively. $\varepsilon_{0}$ is the vacuum dielectric constant. The n-th order electron polarization is

$$
P^{(n)}(t)=\frac{1}{V} \operatorname{Tr}\left(\rho^{n} e z\right)
$$

Where $\operatorname{Tr}$ represents the trace of the matrix, that is, the sum of the diagonal elements of the matrix $\rho^{n} e z$, and $V$ represents the volume of the system. Using the density matrix form and iterative method, the optical rectification coefficient of the system is obtained [30,31]

$$
\chi_{0}^{2}=\frac{4 e^{3} \sigma_{v}}{\varepsilon_{0} \hbar^{2}} M_{01}^{2} \delta_{01} \frac{\omega_{10}^{2}\left(1+\frac{\Gamma_{2}}{\Gamma_{1}}\right)+\left(\omega^{2}+\Gamma_{2}^{2}\right)\left(\frac{\Gamma_{2}}{\Gamma_{1}}-1\right)}{\left[\left(\omega_{10}-\omega\right)^{2}+\Gamma_{2}^{2}\right]\left[\left(\omega_{10}+\omega\right)^{2}+\Gamma_{2}^{2}\right]}
$$

Where $\sigma_{v}$ represents the electron density in the relative quantum dot, $M_{i j}=\left|\left\langle\psi_{i}|z| \psi_{j}\right\rangle\right|$ is a non-diagonal matrix element, $\delta_{01}=\left|M_{22}-M_{11}\right|, \omega_{i j}=\frac{E_{i}-E_{j}}{\hbar}$ is the transition frequency, $\Gamma_{k}=\frac{1}{T_{k}}$ is the damping term related to the life of electrons in the transition.

\section{Results and discussion}

In this section, the following systematic analysis will be made on the optical rectification coefficients in the quantum dots tuned under the action of a vertical magnetic field. Mainly discuss the influence of vertical magnetic field $\mathrm{B}$, hydrostatic pressure $\mathrm{P}$, quantum dot radius $\mathrm{R}$, temperature $\mathrm{R}$, harmonic restraint frequency $\omega_{0}$, limit potential depth $V_{0}$ and dimensionless parameter $\xi$ on the OR coefficient. The parameters used in the calculation are as follows: $\sigma_{v}=5 \times 10^{24} \mathrm{~m}^{-3}, \varepsilon_{0}=$ $8.85 \times 10^{-12} \mathrm{Fm}^{-1}, \hbar=1.055 \times 10^{-34} \mathrm{~J} \cdot \mathrm{s}, m=9.1 \times 10^{-31} \mathrm{~kg}, e=1.602 \times 10^{-19} \mathrm{C}, \Gamma=5 \times$ $10^{12} \mathrm{~Hz}[32,33]$.

As shown in Figure 1, the influence of the vertical magnetic field on the optical rectification coefficient of the tuned quantum dot is discussed. Take $\mathrm{P}=12 \mathrm{kbar}, \mathrm{T}=400 \mathrm{k}, \xi=1, \omega_{0}=$ $30 \times 10^{12} \mathrm{THz}, \mathrm{R}=12 \mathrm{~nm}, \mathrm{~V}_{0}=300 \mathrm{meV}$. The relationship between the optical rectification coefficient and the energy of the incident photon under the action of different vertical magnetic fields is made. The vertical magnetic field strength is respectively taken as $B=4 、 6 、 8 \mathrm{~T}$. It can be seen from the figure that as the intensity of the magnetic field increases, the resonance peak of the OR coefficient moves toward the high-energy region, that is, "blue shift".The main reason is that with the increase of the magnetic field, the quantum confinement effect weakens, and the energy level interval $E_{i j}$ increases accordingly, which leads to the blue shift behavior. At the same time, it can also be found that as the photon energy increases, the peak value of the OR coefficient gradually decreases. This phenomenon is closely related to the density matrix. It can be concluded from formula (29) that the decrease of the density matrix $M_{i j}$ will affect the change of the OR coefficient. 


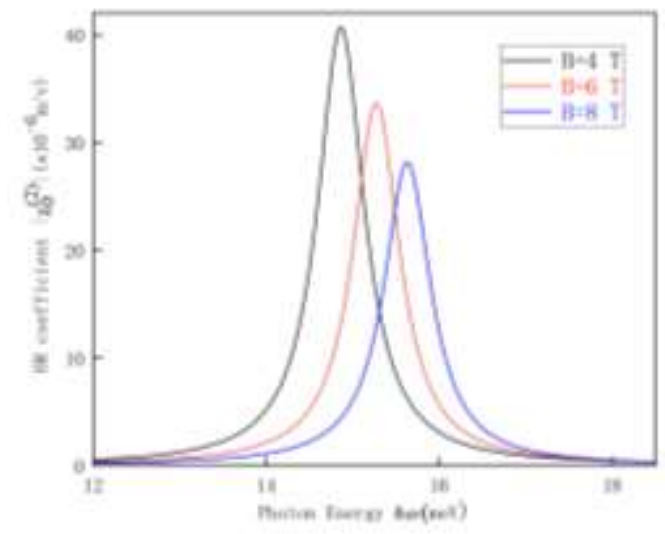

Figure 1 When $B=4,6,8 \mathrm{~T}$, the variation curve of optical rectification coefficient with incident photon energy

As shown in Figure 2, the influence of hydrostatic pressure $\mathrm{P}$ on the optical rectification coefficient in the tuned quantum dot is discussed. Take $\mathrm{B}=6 \mathrm{~T}, \mathrm{~T}=400 \mathrm{k}, \xi=1, \omega_{0}=30 \times$ $10^{12} \mathrm{THz}, \mathrm{R}=12 \mathrm{~nm}, \mathrm{~V}_{0}=300 \mathrm{meV}$. The relationship between the optical rectification coefficient and the energy of the incident photon under the action of different hydrostatic pressures is made, and the hydrostatic pressures are respectively taken as $\mathrm{P}=0,12$, and $24 \mathrm{kbar}$. It can be seen from the figure that as the hydrostatic pressure $\mathrm{P}$ increases, the resonance peak of the $\mathrm{OR}$ coefficient moves toward the low-energy region, that is, a "red shift" occurs. The physical reason is that the hydrostatic pressure has an effect similar to reducing the constraint, which increases the effective mass of electrons, which in turn leads to a decrease in the strength constraint. The physical origin of this "redshift" behavior is that the energy difference between the ground state and the first excited state changes by changing the hydrostatic pressure, The energy difference $E_{10}$ between the ground state and the first excited state decreases with the increase of hydrostatic pressure. Therefore, by increasing the hydrostatic pressure, the resonance peak of the OR coefficient moves to the low-energy region and "redshifts".At the same time, it can be observed from the graph of the change of the OR coefficient with the incident photon energy that the peak value of the OR coefficient will decrease with the increase of the hydrostatic pressure. These effects can be easily used by the separation energy $E_{21}$ between the first and second states. To explain. According to formulas (2) and (3), this is because the effective mass of electrons increases with the increase of external pressure. Therefore, the "red shift" of photon energy with increasing pressure can be used as an adjustment parameter to obtain the required transition energy.

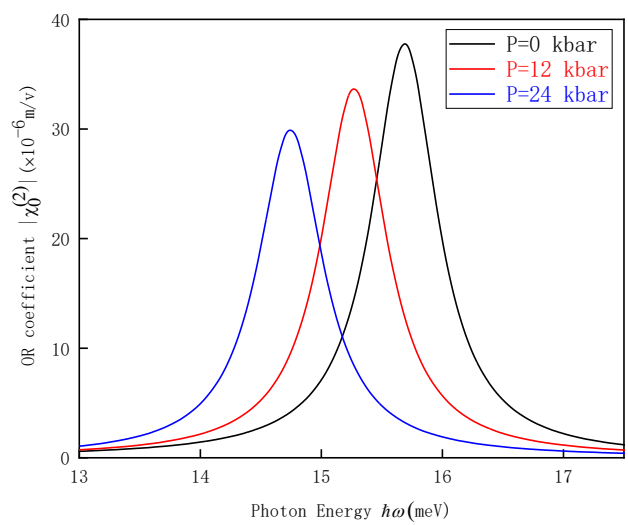

Figure 2 When $\mathrm{P}=0,12,24 \mathrm{kbar}$, the variation curve of optical rectification coefficient with incident photon energy 
As shown in Figure 3, the influence of the radius of the quantum dot on the optical rectification coefficient in the tuned quantum dot is discussed. Take $\mathrm{B}=6 \mathrm{~T}, \mathrm{~T}=400 \mathrm{k}, \xi=1$, $\omega_{0}=30 \times 10^{12} \mathrm{THz}, \quad \mathrm{P}=12 \mathrm{kbar}, \mathrm{V}_{0}=300 \mathrm{meV}$. A graph of the relationship between the OR coefficient and the energy of the incident photon under the action of different quantum dot radii is made. The quantum dot radii are respectively taken as $\mathrm{R}=10,12$, and $14 \mathrm{~nm}$. It can be seen from the figure that as the radius of the quantum dot increases, the resonance peak of the OR coefficient moves toward the low-energy region, that is, a "red shift" occurs. It can be seen from formula (4) that an increase in the radius of the quantum dot will cause the modified Gaussian potential to decrease, resulting in a smaller quantum confinement effect, and the energy level spacing of the confined electrons in the quantum dot will decrease, so the resonance peak of the OR coefficient moves to the lower energy direction, that is, a "red shift" occurs.. At the same time, it can be seen from the figure that as the radius increases, the peak value of the OR coefficient increases. This is because the matrix element $M_{i j}$ increases with the increase of the quantum dot radius $\mathrm{R}$, and as the radius increases, the wave function overlap phenomenon also increases. It is becoming more and more obvious that this is the reason for this physical property.

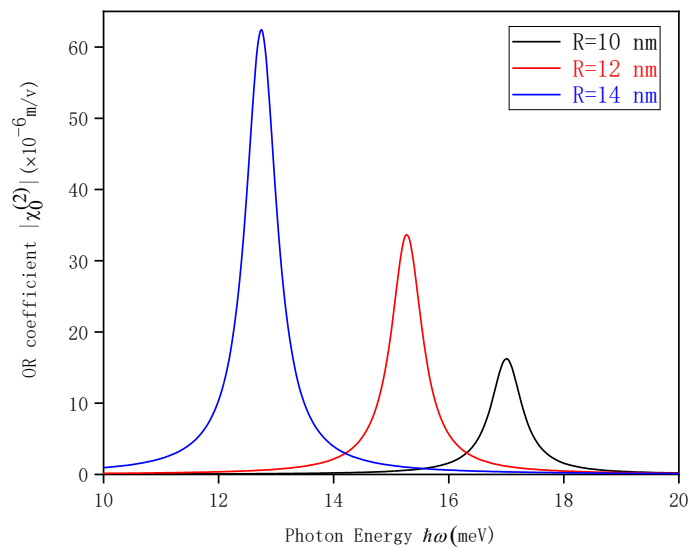

Fig. 3 When $\mathrm{R}=10,12,14 \mathrm{~nm}$, the variation curve of optical rectification coefficient with incident photon energy

As shown in Figure 4, the influence of temperature on the optical rectification coefficient in the tuned quantum dot is discussed. Take $B=6 \mathrm{~T}, \mathrm{R}=12 \mathrm{~nm}, \xi=1, \omega_{0}=30 \times 10^{12} \mathrm{THz}, \mathrm{P}=$ $12 \mathrm{kbar}, \mathrm{V}_{0}=300 \mathrm{meV}$. A graph of the relationship between the third harmonic generation coefficient and the incident photon energy under different temperatures is made, and the temperatures are respectively taken as: $\mathrm{T}=300,400,500 \mathrm{~K}$. It can be seen from the figure that as the temperature increases, the resonance peak of the OR coefficient moves toward the high-energy region, that is, "blue shift". From formulas (2) and (3), it can be seen that as the temperature increases, the effective mass of the electron decreases, and the energy difference between the ground state and the first excited state becomes larger. Therefore, the photon energy moves toward the high-energy region as the temperature increases. It can be seen from the figure that the peak value of the OR coefficient increases with the increase of temperature. This is because with the increase of temperature, the confinement potential and the effective mass of electrons $\mathrm{m}^{\wedge *}$ decrease, and then the separation between the energy of the first and second states increases. 


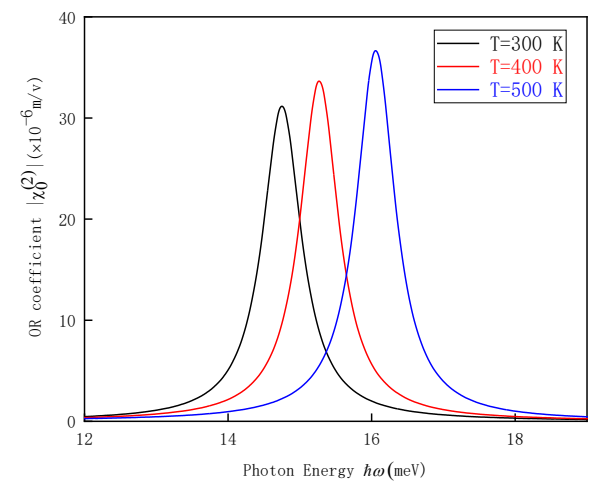

Figure 4 When $\mathrm{T}=300,400,500 \mathrm{~K}$, the variation curve of optical rectification coefficient with incident photon

energy

As shown in Fig. 5, the influence of limiting the potential depth V_0 on the optical rectification coefficient in the tuning quantum dot is discussed. Take $B=6 \mathrm{~T}, \mathrm{R}=12 \mathrm{~nm}, \xi=$ $1, \omega_{0}=30 \times 10^{12} \mathrm{THz}, \mathrm{P}=12 \mathrm{kbar}, \mathrm{T}=400 \mathrm{~K}$. The relationship between the OR coefficient and the incident photon energy under the action of different limiting potential depths is made, and the limiting potential depths are respectively taken as: $V_{0}=250,300,350 \mathrm{meV}$. It can be seen from the figure that as the limiting potential depth $\mathrm{V}_{0}$ increases, the resonance peak of the OR coefficient moves toward the high-energy region, that is, "blue shift". As the limiting potential depth $V_{0}$ increases, the energy level interval increases, which leads to blue shift behavior. It can be seen from the figure that the peak value of the THG coefficient increases with the increase of the limit potential depth $V_{0}$. According to formulas (1), (11) and (29), as $V_{0}$ increases, the energy level interval $E_{i j}$ increases, so that the peak value of the OR coefficient shows an increasing trend.

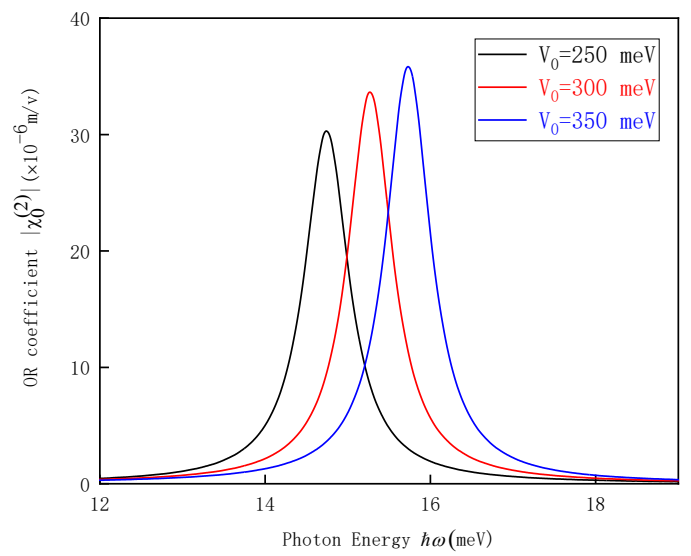

Fig. 5 Variation curve of optical rectification coefficient with incident photon energy when $V_{0}=$ $250,300,350 \mathrm{meV}$

As shown in Figure 6, the influence of the confinement wave frequency on the optical rectification coefficient in the tuned quantum dot is discussed. Take $\mathrm{B}=6 \mathrm{~T}, \mathrm{R}=12 \mathrm{~nm}, \xi=$ $1, V_{0}=300 \mathrm{meV}, \mathrm{P}=12 \mathrm{kbar}, \mathrm{T}=400 \mathrm{~K}$. The relationship between the optical rectification coefficient and the incident photon energy under the action of different confinement wave frequencies is made. The confinement wave frequency $\omega_{0}$ is taken as follows: $\omega_{0}=$ 25、30、35THz. It can be seen from the figure that as the frequency of the confinement wave increases, the resonance peak of the OR coefficient moves toward the high-energy region, that is, 
"blue shift". The physical source of this displacement is the quantum confinement effect in these nanostructures, which causes energy level separation, and the weaker the confinement effect, the more obvious the separation phenomenon. It can be seen from formula (1) that with the increase of $\omega_{0}$, the binding potential $V_{1}(r)$ will also increase, and the quantum confinement effect will increase, and the energy level spacing of the confined electrons in the quantum dot will increase, so the resonance peak of the OR coefficient moves to the high-energy direction, that is, "blue shift" occurs.

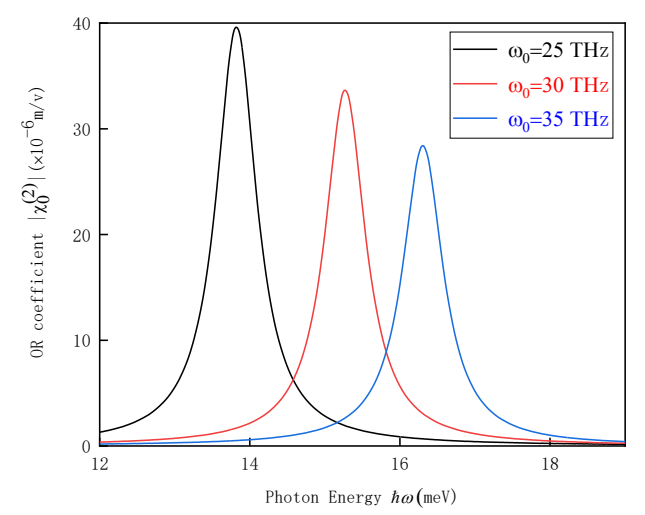

Fig. 6 When $\omega_{0}=25 、 30 、 35 \mathrm{THz}$, the variation curve of optical rectification coefficient with incident photon energy

As shown in Figure 7, the influence of the dimensionless parameter $\xi$ on the coefficient of light rectification in the tuned quantum dot is discussed. Take $B=6 \mathrm{~T}, \mathrm{R}=12 \mathrm{~nm}, \xi=1, \omega_{0}=30 \times$ $10^{12} \mathrm{THz}, V_{0}=300 \mathrm{meV}, \mathrm{T}=400 \mathrm{~K}$.The relationship between the light rectification coefficient and the energy of the incident photon under the action of different parameters is made, and the dimensionless parameter $\xi$ is respectively taken as: $\xi=0.5,1,1.5$. It can be seen from the figure that the increase in the parameter $\xi$ leads to an increase in the peak value of the OR coefficient.". From formula (1), it can be seen that the main reason is that as the parameter value increases, the binding potential $V_{1}(r)$ will also increase, resulting in an increase in the energy level interval, which leads to a blue shift behavior. At the same time, we can also find that as the value of the parameter $\xi$ increases, the peak value of the OR coefficient gradually increases. This is because the increase of the parameter $\xi$ will cause the energy difference between different energy levels to become larger. According to formula (29), The large energy difference makes $\left(\omega-E_{10}+i \Gamma_{10}\right)\left(2 \omega-E_{20}+\right.$ $\left.i \Gamma_{20}\right)\left(3 \omega-E_{30}+i \Gamma_{30}\right)$ decrease, and the peak value of OR coefficient will increase obviously.

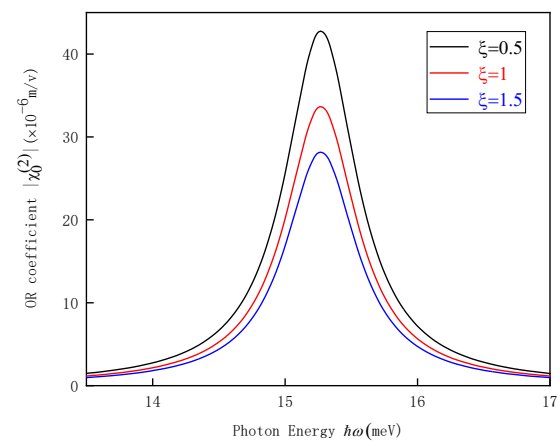

Fig. 7 When $\xi=0.5,1,1.5$, the variation curve of optical rectification coefficient with incident photon energy

\section{Conclusion}


The energy level and wave function are derived by the effective mass approximation and parabolic approximation, and then the density matrix theory and iterative method are used to obtain the analytical expression of the optical rectification coefficient. Numerical calculations show that the peak value of the OR coefficient increases with the increase of the dimensionless parameter and the radius of the quantum dot, and decreases with the increase of the vertical magnetic field. In addition, the increase of the limit potential depth, temperature and confinement wave frequency will cause the OR coefficient resonance peak to move to the high energy direction, and the increase of hydrostatic pressure will cause the OR coefficient resonance peak to move to the low energy direction. Finally, I sincerely hope that our theoretical research results will have a certain impact on the development of nonlinear optics and the renewal of optoelectronic equipment.

\section{Acknowledgments}

We would like to thank the National Natural Science Foundation of China (Grant Nos. 51702003, 61775087, and 11674312), the Provincial Foundation for Excellent Top Talents of Colleges and Universities of Anhui Province of China(Grant No. gxgwfx2019016), the Anhui Provincial Natural Science Foundation, China (Grant Nos.1808085ME130 and 1508085QF140), University Outstanding Young Talents Support Program Fund (Grant No. gxyqZD2018039). 


\section{References}

[1] BASKOUTAS S, PASPALAKIS E, TERZIS A F 2006. Effects of excitons in nonlinear optical rectification in semiparabolic quantum dots. Physical Review B [J], 74.

[2] SARI H, YESILGUL U, UNGAN F, et al. 2017. Intense laser field effects on the intersubband optical absorption and refractive index change in the $\delta$-doped GaAs quantum wells. Chemical Physics [J], 487: 11-15.

[3] HASHEMI P, SERVATKHAH M, POURMAND R 2021. The effect of rashba spin-orbit interaction on optical far-infrared transition of tuned quantum dot/ring systems. Optical and Quantum Electronics [J], 53.

[4] OZTURK O, OZTURK E, ELAGOZ S 2019. Depending on the intense laser field of the nonlinear optical rectification, second and third harmonic generation in asymmetric parabolic-step and inverse parabolic-step quantum wells. Physica Scripta [J], 94.

[5] KARIMI M J, REZAEI G 2011. Effects of external electric and magnetic fields on the linear and nonlinear intersubband optical properties of finite semi-parabolic quantum dots. Physica B: Condensed Matter [J], 406: 4423-4428.

[6] HU M, GUO K, ZHANG Z, et al. 2017. The effect of position-dependent mass on nonlinear optical absorption coefficients and refractive index changes in a quantum well. International Journal of Modern Physics B [J], 31.

[7] YU Y-B, ZHU S-N, GUO K-X 2005. Exciton effects on the nonlinear optical rectification in one-dimensional quantum dots. Physics Letters A [J], 335: 175-181.

[8] HASSANABADI H, LIU G, LU L 2012. Nonlinear optical rectification and the second-harmonic generation in semi-parabolic and semi-inverse squared quantum wells. Solid State Communications [J], 152: 1761-1766.

[9] LI B, GUO K-X, ZHANG C-J, et al. 2007. The second-harmonic generation in parabolic quantum dots in the presence of electric and magnetic fields. Physics Letters A [J], 367: 493-497.

[10] ZHANG Z-H, GUO K-X, CHEN B, et al. 2010. Theoretical studies on the optical absorption coefficients and refractive index changes in parabolic quantum dots in the presence of electric and magnetic fields. Superlattices and Microstructures [J], 47: 325 334.

[11] BOUZAïENE L, BEN MAHRSIA R, BAIRA M, et al. 2013. Hydrostatic pressure and temperature effects on nonlinear optical rectification in a lens shape InAs/GaAs quantum dot. Journal of Luminescence [J], 135: 271-275.

[12] GANGULY J, SAHA S, BERAA, et al. 2016. Modulating optical rectification, second and third harmonic generation of doped quantum dots: Interplay between hydrostatic pressure, temperature and noise. Superlattices and Microstructures [J], 98: 385-399. [13] KHORDAD R 2013. Effect of magnetic field on linear and nonlinear optical properties in a parabolic cylindrical quantum dot. Journal of Optics [J], 42: 83-91.

[14] DAHIYA S, LAHON S, SHARMA R 2020. Effects of temperature and hydrostatic pressure on the optical rectification associated with the excitonic system in a semi-parabolic quantum dot. Physica E: Low-dimensional Systems and Nanostructures [J], 118.

[15] ZHANG L, LI X, LIU X, et al. 2021. The influence of second-harmonic generation under the external electric field and magnetic field of parabolic quantum dots. Physica B: Condensed Matter [J], 618.

[16] KARABULUT Í, DUQUE C A 2011. Nonlinear optical rectification and optical absorption in GaAs-Ga1-xAlxAs double quantum wells under applied electric and magnetic fields. Physica E: Low-dimensional Systems and Nanostructures [J], 43: $1405-$ 1410.

[17] HIEN N D, DUQUE C A, FEDDI E, et al. 2019. Magneto-optical effect in GaAs/GaAlAs semi-parabolic quantum well. Thin Solid Films [J], 682: 10-17.

[18] RESTREPO R L, KASAPOGLU E, SAKIROGLU S, et al. 2017. Second and third harmonic generation associated to infrared transitions in a Morse quantum well under applied electric and magnetic fields. Infrared Physics \& Technology [J], 85: 147-153. [19] YUAN J-H, CHEN N, ZHANG Y, et al. 2016. Electric field effect on the second-order nonlinear optical properties in semiparabolic quantum wells. Physica E: Low-dimensional Systems and Nanostructures [J], 77: 102-107. 
[20] YOU J-F, ZHAO Q, ZHANG Z-H, et al. 2019. The effect of temperature, hydrostatic pressure and magnetic field on the nonlinear optical properties of AlGaAs/GaAs semi-parabolic quantum well. International Journal of Modern Physics B [J], 33.

[21] REZAEI G, VASEGHI B, TAGHIZADEH F, et al. 2010. Intersubband optical absorption coefficient changes and refractive index changes in a two-dimensional quantum pseudodot system. Superlattices and Microstructures [J], 48: 450-457.

[22] MO S, GUO K, LIU G, et al. 2020. Exciton effect on the linear and nonlinear optical absorption coefficients and refractive index changes in Morse quantum wells with an external electric field. Thin Solid Films [J], 710.

[23] KUMAR M, LAHON S, JHA P K, et al. 2014. Spin-orbit interaction effect on nonlinear optical rectification of quantum wire in the presence of electric and magnetic fields. Physica B: Condensed Matter [J], 438: 29-33.

[24] LI B, GUO K-X, LIU Z-L, et al. 2008. Nonlinear optical rectification in parabolic quantum dots in the presence of electric and magnetic fields. Physics Letters A [J], 372: 1337-1340.

[25] HU M, GUO K, YU Q, et al. 2018. Polaron effects on nonlinear optical refractive index changes in semi-exponential quantum wells. Opt Lett [J], 43: 3550-3553.

[26] PAL S, GHOSH M, DUQUE C A 2019. Impurity related optical properties in tuned quantum dot/ring systems. Philosophical Magazine [J], 99: 2457-2486.

[27] STEVANOVIĆ L, FILIPOVIĆ N, PAVLOVIĆ V 2019. Effect of magnetic field on absorption coefficients, refractive index changes and group index of spherical quantum dot with hydrogenic impurity. Optical Materials [J], 91: 62-69.

[28] RASTEGAR SEDEHI H R, KHORDAD R 2021. Magnetocaloric effect, magnetic susceptibility and specific heat of tuned quantum dot/ring systems. Physica E: Low-dimensional Systems and Nanostructures [J], 134.

[29] UNGAN F 2017. Effects of applied electric and magnetic fields on the nonlinear optical rectification and second-harmonic generation in a graded quantum well under intense laser field. The European Physical Journal B [J], 90.

[30] YESILGUL U, UNGAN F, SAKIROGLU S, et al. 2014. Effect of intense high-frequency laser field on the linear and nonlinear intersubband optical absorption coefficients and refractive index changes in a parabolic quantum well under the applied electric field. Journal of Luminescence [J], 145: 379-386.

[31] YU Q, GUO K, HU M, et al. 2018. Study on the optical rectification and second-harmonic generation with position-dependent mass in a quantum well. Journal of Physics and Chemistry of Solids [J], 119: 50-55.

[32] ZHAI W 2014. A study of electric-field-induced second-harmonic generation in asymmetrical Gaussian potential quantum wells. Physica B: Condensed Matter [J], 454: 50-55.

[33] KARABULUT İ, MORA-RAMOS M E, DUQUE C A 2011. Nonlinear optical rectification and optical absorption in GaAsGa1-xAlxAs asymmetric double quantum wells: Combined effects of applied electric and magnetic fields and hydrostatic pressure. Journal of Luminescence [J], 131: 1502-1509. 